\title{
Estado de La Doctrina y la Jurisprudencia en la Responsabilidad Medica
}

\author{
Celia Weingaxten
}

Professora de Direito Civil da Faculdade de Direito da

Universidade de Buenos Aires, Argentina

\section{Introducción.}

1 ejercicio de la medicina, 14 historicamente, se asentó sobre bases paternalistas, el médico asumía

\begin{tabular}{|c|}
\hline SUMARIO \\
1. Introducción. \\
2. Caracterización del contrato médico \\
como de adhesión. \\
3. El deber de información. \\
4. El acto médico: la discresionalidad ci- \\
entífica y su adaptación a las circunstancias \\
contextuales del paciente. Su relación con \\
el daño. \\
5.Sistemática probatoria. Actividad \\
procesal de las partes. \\
6. La obligación de indemnidad de los \\
establecimientos asistenciales. \\
\hline
\end{tabular}
un papel casi sacerdotal, estableciendose con el paciente una especie de relación divina y científicamente incuestionable, en cuyas manos se encontraban la vida o la muerte del individuo.

El contrato implicaba entonces una situación de sumisión del paciente en la toma de decisiones respecto de su cuerpo y la dirección de la intervencion medical; todo quedaba librado a la voluntad del medico. Su caracter sagrado y venerable le conferia una situacion de privilegio, autoridad e impunidad, que escapaba a todo juicio de responsabilidad.

Ningun lugar cabía para la negligencia, ni siquiera para el error, y tampoco ninguna diferencia había entonces entre el verdadero supuesto de incurabilidad o aquellos de la mala praxis. La respuesta de la sociedad y de la victima era la resigna- 
ción, el resultado inalcanzado era simplemente calificado como un desenlace inevitable que el paciente debia aceptar como un hecho fatal, como acepta la enfermedad misma.

Hoy dia la responsabilidad civil del medico transita por caminos diferentes. Desde esa confianza ciega y falta de cuestionamiento científico-social de entonces, se inicia un camino inverso de desmitificación y quiebra de la inmunidad del que los médicos han gozado hasta no hace mucho tiempo, aun cuando algunos resabios de esa epoca lamentablemente perduran.

Distintos acontecimientos han signado estos nuevos rumbos, y otros son los escenarios que han contribuido a la evolucion de la responsabilidad medica.

Con el vertiginoso desarrollo de la ciencia y tecnología médica operada en la segunda mitad de este siglo, la medicina se vi "sustentada por un mayor aporte técnico y científico, lo que implic" la sujeción del accionar médico a esos principios y métodos establecidos por la ciencia médica. Ello marca un transito que va de una concepcion autoritaria a otra de tipo "cientificista" de la prestación médica.

A partir de la consagracion de los derechos del Hombre, comienza un proceso de afianzamiento de los derechos individuales, que luego evolucionaron hacia el desarrollo de los derechos personalisimos. De ellos se derivan un amplisimo reconocimiento de otros derechos de la persona, otorgando proteccion a aspectos funda- mentales ignorados por nuestro Codigo del siglo pasado, colocando hoy al hombre en el centro del sistema jurídico.

Entre ellos se ubica el derecho a la información, que se incardina en el marco mas amplio de la libertad, $y$ tiene una significación instrumental pues a través de ella se posibilita el ejercicio de otros derechos que involucran distintos aspectos de los pacientes, tales como el de disposicion del propio cuerpo, su libertad personal, de decidir su vida, etc.

En otro plano, aparece la figura del consumidor, como sujeto necesitado de primordial atención y protección por el derecho, el que se proyecta en diversos ambitos reclamando esa actitud tuitiva, jerarquizado luego por la reforma constitucional de 1994 .

Sin duda se asiste a una evolucion de trascendencia en este campo, de la que pretendemos dar cuenta. Dado los limites de este trabajo, solo habremos de analizar algunos de los aspectos mas opinables de la responsabilidad medica, para tratar de hacer un aporte mas hacia su esclarecimiento.

\section{Caracterización del contrato medico como de adhesion.}

Se trata de una relacion que pone de manifiesto una desigual situacion juridica, psicologica y cientifica de los contratantes, que rompe con el modelo contractual paritario concebido por Velez Sarfield, como un acuerdo que se celebra entre iguales que poseen similar poder de negociacion, en libertad.

1. Weingarten Celia en Ghersi Carios "Responsabilidades Profesionales", vol. 1, pag. 59 y sgtes, Ed. Astrea. Bs. As. 1995
Uno de los rasgos distintivos de la contratación profesional es el "caracter cientifico intelectual" de la prestación, en el que prevalece el saber del profesional en un campo del conocimiento ajeno al paciente.

Dado este caracter eminentemente cientifico que presenta el contrato, desaparece la libertad de configuracion de su contenido, al carecer el paciente de los conocimientos cientificos necesarios.

Se suma a ello, el propio estado de salud del paciente que reclama asistencia ${ }^{2}$. El individuo enfermo, temeroso por su salud y quizas por su vida, se presenta con frecuencia como "debil, desvalido, dependiente y facilmente manipuleable" por el profesional, en cuya actuacion confia, y ello determina que se ponga en manos de su accion curativa, lo que provoca una conducta de disposición y sumisión del paciente frente a su medico. Es sabido como cualquier enfermedad, por mínima que sea, sume a cualquier sujeto en un estado de indefensión y debilidad psíquica, colocando al profesional en una situación de poder. ${ }^{3}$

Este condicionamiento subjetivoobjetivo, se coloca en situacion de "conexidad" con otro factor de trascenden- cia: "la influencia psicologica de la confianza en el profesional" en el proceso de curacion, no solamente a partir de sus calidades especificas y la consecuencte confianza que genera, que influye decisivamente en la eficacia terapeutica, sino que ademas se convierte, por distintos mecanismos psicologicos, en parte esencial del proceso curativo, ya que es quien promueve y dirige la curación del enfermo. ${ }^{4}$

Esta posición asimétrica de la relación profesional, en la que el paciente asume el rol del mas debil frente al poder cientifico del facultativo desequilibrante de la relacion contractual, es la que nos permite encuadrarla dentro de la estructura de adhesion de modalidad individual.

El contrato no es ya el resultado de una decision libre y voluntaria que el consentimiento implica, y poco tiene que ver con la igualdad de los sujetos contrtantes que la teoria contractualista postula, sino que es la expresion de la superioridad socio-cultural del facultativo sobre el paciente. No hay entonces un verdadero consentimiento, sino que el paciente solo expresa su asentimiento, ${ }^{5}$ y por lo tanto resulta revisable judicialmente.

Dado que el contrato medico se realiza por la estructura de la adhesion, en-

2. Weingarten Celia, Contrato y Responsabilidad Medica: El deber de informacion y el consentimiento informado (Una visión crítica), Jurisprudencia Argentina, Semanario Marzo 19 de 1997.

3. Consult. Galbraith, John "La anatomía del poder", Ed. Plaza, Janes, Madrid 1985.

4. Jones, Jesse E. v/ Georgetown Universitu Hospital 1964, Corte Federal de Apelaciones del Circuito de Columbia: "Los esquemas normales jurídicos de la relación médico paciente están basados en estructuras jurídicas contractuales o comerciales que se ven debilitadas en situacion de urgencia, de vida o muerte. No est claro que el paciente pueda dirigir el tratamiento aplicable bajo debilitadas en situacion de urgencia, de vida o muerte. No est
limitaciones de las que se deriva irremediablemente la muerte".

5. Lipari Nicolo, "Derecho Privado. Un ensayo para la enseñanza", Ed. Publicaciones del Real Colegio de España, Zaragoza, 1989, pag. 336 "No se trata de un acto volitivo, sino un hecho meramente cognoscitivo de una de las partes. En otros términos, faltaría en tales hipótesis, la formación de un verdadero y propio acuerdo en sentido técnico jurídico". 
tendemos que son alcanzados por la regulacion de la Ley de Defensa del Consumidor Nro. 24.240.

Sin embargo, dicha normativa establece ciertas exclusiones, entre ellas la de los profesionales liberales que requieren titulo universitario y matriculacion, salvo la publicidad que se haga de su ofrecimiento.

Siendo la nota tipificante de las prestaciones medicas la conformacion por contratos de adhesion, entendemos que solo quedarian excluidos de la aplicacion de la ley los contratos con profesionals liberales cuando son de negociacion individual y no cuando esos contratos se celebren a traves de los contratos de adhesion, que son la mayoria.

Por ende, esa contradiccion que la ley presenta debe resolverse hacia su aplicacion, confirmando asi su finalidad teleológica, que es la de proteger a quienes se encuentran en condiciones de minusvalía genética de esas estructuras negociales.

\section{El deber de información.}

Frente a esta notoria desigualdad que las partes exhiben, en especial el mayor conocimiento cientifico del profesional, el derecho de informacion adquiere especial significacion pues tiene como finalidad morigerar ese desequilibrio genetico-funcional, colocando en manos del paciente una "herramienta de control" para limitar el poder cultural desequilibrante de los profesionales.

La relevancia de la informacion ha justificado su consagración constitucional,

\footnotetext{
6. Diaz Picazo, Luis, "Fundamentos del derecho civil patrimonial", Ed. Tecnos, Madrid 1970,pag. 110: "La voluntad en el consentimiento presupone un perfecto conocimiento del alcance del negocio y la libertad de querer sus consecuencias".
}

Revista da Faculdade de Direito da UFRGS, v. 13, 1997 en su reforma de 1994 que, al reconocer el derecho primario o basico del derecho de acceso al consumo, se incluye una serie de derechos sustanciales, entre ellos a la proteccion de su salud, seguridad, y especificamente, el derecho a obtener informacion adecuada y veraz.

En materia de contratacion medica, algunas disposiciones aisladas establecen tales deberes informativos, as! por ejemplo en materia de transplantes, sida, y la ley de ejercicio de la medicina Nro. 17.132 que al requerir del paciente conformidad por escrito en las operaciones mutilantes, conlleva implicitamente la obligacion de información previa para permitirle ejercer esa facultad.

A partir de tales prescripciones que son aplicables analogicamente, tanto la doctrina como la jurisprudencia son coincidentes en la existencia de un deber legal y contractual del profesional de informar.

Sin embargo, el tema presenta aristas que exhiben una notable complejidad, como es lo inherente al denominado "consentimiento informado", que parte de la doctrina sostiene y que no compartimos, en especial cuando se lo pretende imponer responsabilidad del acto medico.

Dado las caracteristicas de la relacion medico-paciente del que antes dabamos cuenta, entendemos que el consentimiento prestado por el paciente se encuentra sumamente restringido por multiples factores $^{6}$, como por ejemplo su falta de conocicomo condicion de licitud o eximente de mientos cientificos para comprender variables terapeuticas, su propia condicion de enfermo, la relacion de dependencia y sumision con respecto al profesional, etc. que condicionan y reducen la capacidad de elegir, decidir, o tomar opciones.

De allí que, como regla general, el consentimiento no tiene la amplitud que estas teorias postulan, porque los pacientes y los medicos no son iguales en cuanto a posesión de informacion ni a su capacidad de controlar las circunstancias en las que se encuentran. Concretamente, una de las partes esta sana y tiene los conocimientos medicos, mientras que la otra esta enferma y no los tiene.

Es cierto que la falta de información puede coaccionar las opciones de una persona; cuanto menos informacion disponga, menos posibilidad tendr para tomar una decisión, y esto en s! y per se es violatorio de los deberes constitucionales y autonomamente potencial generador de daño.

Aun disponiendo de informacion, sin embargo, no siempre el paciente es capaz de manejar y comprender la informacion que se le suministra, ni tampoco puede captar los hechos relevantes de su estado, ni de las posibles alternativas terapeuticas, o comprender el significado de un traamiento o técnica propuesta.

En la doctrina y jurisprudencia extranjera, la eficacia jurídica del consentimiento es mas restringido, y ha expresado

Beaychamps, Tom L. y McCullough, Laurence B "Etica Médica. Las responsabilidades morales de los médicos", pag.46 Ed.Labor, Buenos Aires 1987

8. Linden, Allen M Canadian Tort Law, Ed. Butterworths, Toronto and Vancouver, 1993, pag. 65. que, "alli donde opere una relacion de dependencia, traducida en una marcada inequitatividad en los poderes respectivos de las partes y de la superioridad del mas fuere sobre el mas debil, el consentimiento no es genuino. Esta situacion de poder se presume en la relacion medico paciente, $y$ esto es lo que hace dificil distinguir la coercion y la manipulacion, de la simple persuasion, o el consejo de la influencia".

La realidad es que el profesional propone, y el paciente, al no estar cientificamente capacitado para comprender el contenido del contrato y tomar decisiones, tan solo se limita a someterse o no al tratamiento que él ha propuesto como el mas adecuado.

Por ende, su "eficacia juridica" es muy limitada, y no constituye una eximente de responsabilidad del profesional, siendo suceptible de ser revisada judicialmente. De otro modo, se correría el peligro de que bajo el "aparente" consentimiento del paciente -generalmente instrumentado en formularios preelaborados-se legitime cualquier practica medica, convirtiendose en un mero mala practica medica.

Es por ello que dadas las limitaciones cientificas y psicologicas del enfermo, entendemos que el contenido de la informacion solo puede versar sobre los riesgos que el tratamiento conlleva, pero no sobre la tecnica o estrategia a seguir, pues esta concierne al contenido del contrato e formalismo, protector corporativo de la 
involucra aspectos cientificos 9 que el paciente, por no comprenderlos adecuadamente, no puede validamente "consentir".

La funcion de la informacion es pues la de dar a conocer los riesgos propios de la estrategia terapeutica que el medico ha conclara, comprensible y adaptada a la situacion socio cultural del paciente ${ }^{10}$, de manera tal que le permita decidir someterse o no al mismo, y esto ultimo con limitaciones.

De acuerdo con lo que venimos exponiendo, cuando el paciente presta su "asentimiento" a un tratamiento o intervencion medica, ello no significa la convalidacion del mismo y queda abierta la posibilidad de su revision. Es decir que no opera como condicion de licitud o eximente de responsabilidad del acto medico. En ese caso, si la estrategia no ha sido la adecuada el facultativo para eximir se de responsabilidad, debera acreditar que ha actuado de forma diligente e idonea y con adecuado fundamento cientifico. Fun damentalmente exige la demostracion que la terapeutica utilizada no ha sido arbitra ria y que dentro de la discrecionalidad cientifica del que goza y las distintas variables cientificas que dispone "ha optado por aquella que cuenta con una mayor idoneidad objetiva", en funcion de la patologia, las circunstancias particulares del enfermo y el desarrollo evolutivo de la medicina (art. 512 CC). siderado como la mas apropiada, en forma

\section{El acto médico: la discresionalidad científica y su adaptación a las circunstancias contextuales del paciente. Su relación con el daño}

Emerge para el medico una obligacion de hacer consistente en el desarrollo de una conducta tecnica y cientificamente adecuada, conforme el estado de la ciencia, tendiente a la obtencion de cierto resultado, aunque este no pueda garantizarse.

En este sentido, la tendencia doctrinaria y jurisprudencial es que el profesional goza de una amplia discresionalidad cientifica ${ }^{11}$ - consecuencia de la independencia intelectual inherente a cualquier profesional liberal -, que le permite, en base a un saber actualizado de la ciencia optar entre distintos caminos alternativos cientificamente posibles e iguales para actuar sobre determinada patologia, previamente diagnosticada.

Una enfermedad puede ser objeto de varios tratamientos, todos con valor terapeuticos, y no puede exigirsele al medico la sujecion a un metodo en particular. Lo importante es que el metodo o tratamiento debe aparecer como objetivamente como uno de los considerados adecuados para esa enfermedad, teniendo en cuenta el estado de la ciencia, es decir que frente a distintas

9. Weber, Max "Law in Economy and Society", Ed. Harvard University, 1954, pag. 323: "El conocimiento es el instrumento de una o mas personas para realizar su propia voluntad contra la voluntad de otro u otros qu participan del mismo acto".

10. Corte Suprema de Buenos Aires, "Kaspar v. Sanatorio Mitre", K 24 XXIV.

11. Weingarten Celia y Ghersi Carlos "La discrecionalidad de la estrategia terapeútica. La responsabilidad del Estado por la seguridad de los pacientes en los hospitales". Voz de la doctrina sobre la Jurisprudencia, Nro.1, año 1997.

Revista da Faculdade de Direito da UFRGS, v. 13, 1997 alternativas terapeuticas cientificas, el medico puede optar por alguna objetivamente idonea de acuerdo a las reglas de la ciencia medica ${ }^{12}$ para casos similares descriptos en obras cientificas, del cual pueden esperarse determinados resultados.

Esta decision es en base a un saber medico objetivo e involucra la utilizacion de terapias habitualmente reconocidas por la ciencia medica, abandonado aquellas practicas o metodos en desuso, extraños o peligrosos, acorde con el desarrollo cientifico (referenciado al espacio geografico de su actuacion).

En un segundo paso, y una vez evaluada la posibilidad objetiva en abstracto, el medico debe producir la adecuacion subjetiva de aquellos caminos cientificos, de acuerdo a la que considere mas adecuada, no solo en funcion a su patologia, sino teniendo presente ademas las circunstancias contextuales condicionantes del paciente, su estado de salud, aspectos economicos, culturales, ambientales etc. que influyen y limitan el accionar profesional.

La mayoria de los conflictos de la responsabilidad medica son causados por la organizacion en la prestacion, insuficiencia de materiales humanos, tecnicos, etc, en la que el medico solo actua como detonador del daño, imputandosele luego una responsabilidad que no le corresponde ${ }^{13}$. De allí que hoy día la responsabilidad del medico debe ser estudiada no aisladamente sino

12. Barreiro Agustin Jorge "La imprudencia punible en la actividad medico-quirúrgica", pag. 47 Ed. Tecnos. Madrid. 1990. 13. Lorenzetti Ricardo, "Nuevos enfoques en la responsabilidad profesional", La Ley, ej. del 5/7/1996.

14. Corte Suprema, Marzo 29-1984, Gonzalez Orono de Leguizamon, Norma M. c/ Federación de Trabajadores Jaboneros y Atines, La Ley 1984-B-389 dentro de la estructura en que desarrolla su profesión, como ya nuestro mas Alto Tribunal pusiera de relieve al diferenciar al profesional aislado del que ejercita en el

Con frecuencia el profesional de la medicina debe rectificar o elegir terapeuticas conforme a los medios economicos directos (coberturas de obras sociales o empresas privadas) o indirectas (capacidad de pago del paciente frente a aparatologia, farmacias, etc.) que luego repercuten en la salud del paciente (causa en el paciente). Ello se encuentra relacionado con los condicionamientos del segmento causal,en la que se deben ponderar algunos otros condicionamientos que tienen relebien no alcanzan a caracterizarse como causa - causa adecuada - y permanece en calidad de condicionante, inciden en la produccion del daño.

Dado estos condicionantes contextuales, la valoracion de la conducta discrecional médica, debe ser efectuada en un doble sentido, no solo en funcion de la ciencia medica actualizada, - en abstracto - sino tambien valorando el estado de cosas y el contexto en que el acto medical es emprendido, con los recursos y posibilidades que haya tenido el profesional en concreto, es decir conforme las circunstancias de lugar, tiempo y persona. (condicionantes contextuales).

Revista da Faculdade de Direito da UFRGS, v. 13, 1997 contexto de una obra social ${ }^{14}$. vancia en la produccion del daño, que si 
De allí que el concepto de discrecionalidad cientifica es sometida ac tualmente a un proceso de revision critica, acorde con un criterio mas realista que des cansa no solo en una valoracion puramente objetiva del acto medico, sino en funcion de esos otros factores que tambien forman parte de esa realidad y de las que no puede prescindirse. El medico forma parte de su entorno al que debe necesariamente adaptarse; sus actos no dependen solo de el sino de circunstancias externas que lo trascien den y condicionan, afectando inclusive su libertad. ${ }^{15}$.

El tema se encuentra ligado con la relacion de causalidad, en la que la valoracion del hecho humano medico asume relevancia para poder establecer cuan do un comportamiento puede causar adecuadamente un resultado para efectuar la atribución de responsabilidad.

Si el medico actua conforme un criterio de discrecionalidad cientifica, tanto en su aspecto objetivo como subjetivo optan do por alguna de las varioables objetivamente idoneas de acuerdo a las reglas de la medicina conforme una adecuacion a la naturaleza de la patologia, caracteristicas del paciente y recursos materiales y economicos existentes, no introduce causalidad alguna para la produccion del daño.

En ese caso el resultado lesivo no puede serle atribuido a su accion $u$ omision, sino a la propia causalidad natural del paciente (su enfermedad) o a condicionantes tecnologicos, economicos, regionales, etc. es decir desplaza la causa y lo coloca fuera del accionar medical.

El paciente asume entonces el riesgo de su enfermedad y tambien el riesgo del tratamiento, realizado con condicionantes inadecuados no dependientes del medico. Ello sin perjuicio de que el daño pueda ser atribuido al ente asistencial, hospital, empresa de medicina, etc.

\section{Sistemática probatoria. Actividad procesal de las partes.}

Una de las cuestiones mas debatidas es sin duda lo atinente a la problematica de la carga probatoria. ${ }^{16}$ La jurisprudencia durante mucho tiempo y casi en forma unanime, sostenía que la prueba estaba en cabeza del acreedor o de la victima que lo alega en toda su extension. Actualmente el tema ha sufrido una importance evolucion y fraccionamiento. El estado actual de la doctrina y la jurisprudencia, es que elmedico debe contribuir con la carga probatoria, y en especial en la prueba del antecedente y la relacion de causalidad, que configuran los hechos centrales para la reparacion del daño.

Quien pretende la reparacion debe alegar, acreeditar y probar el hecho genera-

15. Martinez García, Jesus Ignacio "La imaginación jurídica", Ed. Debate, Madrid 1992, pag. 129: "La teoría de sistemas reorienta el principio de acción clasicamente ligado al sujeto aislado y lo sustituye por la perspectiva de sistemas sociales de acción.La subjetividad del individuo racional y todo el aparato conceptual abstracto ligado a ella - decisión, intenció, fines, elección de medios apropiados o no, previsión de consecuencias - aparece reformulado. El problema del orden social ya no se plantea en términos de una convergencia de voluntades, sino de una construccion social de estructuras cuyo último elemento es la acción

16. Weingarten Celia, Ghersi Carlos, "Responsabilidad medica: La doctrina en casación de la Suprema Corte de la Provincia de Buenos Aires, Jurisprudencia Argentina T 1993-II-891.

Revista da Faculdade de Direito da UFRGS, v. 13, 1997

dor, la existencia del daño, y estalecer que esta "consecuencia" ha sido generada por el "hecho tal cual lo reconstruyó".

A su vez, el demandado tiene que realizar una conducta procesal "activa de colaboracion" - porque es de su interes en la reconstruccion historica del hecho causal, y "destructiva" en cuanto a ligazóncausalidad - de aquel hecho con el daño. Es decir, debe alegar y probar que el daño est fuera de su accionar (vg. caso fortuito, fuerza mayor, culpa de un tercero por el cual no debe responder,etc.); o bien atacando la nor reparabilidad del daño, vg. el daño era previsible pero inevitable.

Desde el enfoque procesal, la necesidad de que ambas partes concurran a la carga de la prueba encuentra su fundamento en la aplicacion de las reglas procesales, correspondiendo no solo a quien afirma un hecho, sino tambien a quien niega su existencia.

En suma, quien alega un hecho debe probarlo, de modo tal que cada parte debe postular y probar los presupuestos de hecho de la norma jurídica que invocare como fundamento de su pretensión, defensa o excepción. Se trata de una carga procesal que aparece no solo como un deber juridico, sino como un imperaivo del propio interes. Es una facultad que se le adjudica a las partes para avalar su interés, e implica que, de no hacerlo, se asumirán las consecuencias.

Este criterio encuentra sustento ademas en el deber de colaboracion y bue-

na fe (art. 1198 CC), de cuyo cumplimiento no queda exento ninguno de los litigantes, mas all de su posición en la litis.

Otro sector doctrinario y jurisprudencial trata de paliar la situacion de la víctima frente a una mejor posicion basado en el privilegio del facultativo, ${ }^{17}$ por tratarse de una vinculacion procesal jurídica que enfrenta a un experto y un profano en la materia (las llamadas cargas probatorias dinamicas). Dada la naturaleza de los hechos que se ventilan, relacionado con aspectos tecnicos cientificos que el paciente no puede conocer ni valorar con la precisión científica, se entiende que sobre el debe pesar la carga de alegar y demostrar el desarollo fáctico-científico de la conducta realizada, evitando colocar al paciente en desigualdad de condiciones.

Conforme lo expresado, corre por cuenta del medico la prueba de su obrar discrecional. En este sentido, debe primero acreditar que la estrategia elegida es una alternativa de la ciencia medica (presupuesto objetivo), y que entre varias opciones posibles vigentes, ha optado por un metodo con sustento cientifico; segundo, que ha realizado un analisis de adaptacion en funcion de la tipologia de la enfermedad y las circunstancias condicionantes externas del caso.

Por consiguiente, la acreditacion del acto discrecional medico conduce a desconectar el resultado dañoso del hecho profesional; el daño no es ya la consecuencia

17. "Tratándose de una acción por daños y perjuicios derivados de un hecho médico, si bien ambas partes deben acreditar sus derechos y desvirtuar sus obligaciones, como forma de colaborar en el logro de una aplicacion justa del derecho, es evidente que la carga pesa sobre quien se encuentra en mejor situacion para producir pruebas, en el caso, el medico, ya que es quien tiene los conocimientos técnicos necesarios para explicar los hechos ocurridos y la vivencia directa de ellos". CNCiv. Sala I Octubre 25-990 ED 141-285. 
de su conducta, con lo cual "outro" sera el "hecho generador", el que puede obedecer a la causalidad de la victima (vg. causa predisponente en el organismo del pacien te), u factores condicionantes externos, vg. causa fortuito o fuerza mayor, o bien a otras situaciones objetivas ajenas al obrar medical o provenientes de la organizacion hospitalaria.

Como vemos, no se trata que el actor deba probar la culpa, ni tampoco implica inversion de la carga probatoria para e profesional, sino que es la verificacion objetiva de la conduta cientifica del facultativo, que de producirse, ubican la causa de daño fuera de su órbita.

De este modo, la reconstruccion de hecho causal cobra trascendencia y no se hace necesario introducirse en el plano de la culpabilidad que aparece como deduccion de logica juridica que el daño se encuentra fuera de su accionar; se impide que se concrete su responsabilidad al no configurarse los presupuestos básicos de la reparación.

\section{La obligación de indemnidad de los \\ establecimientos asistenciales}

Los establecimientos asistenciales asumen frente al enfermo una obligación tácita de indemnidad que le asegura la prestación de un adecuado servicio, preservándolo de eventuales fallas del sistema.
La tendencia actual es la objetivacion de la responsabilidad de los establecimientos asistenciles, ya sean publicos o privados. En este sentido, tanto la doctrina como la jurisprudencia es la de interpretar en forma mas restrictiva los supuestos de culpa del medico que presta servicios en los Hospitales Publicos, pero a la vez amplia considerablemente la responsabilidad de estas instituciones publicas en terminos objetivos, sobre la idea de una deficiente organizacion del servicio.

Frente a este "deficiente funcionamiento del servicio" el Estado responde directa y objetivamente, pues hace a su propia funcion, y no a la actuacion del profesional o dependiente pues parte de una situacion objetiva de falta o deficiencia del servicio que el Estado por mandato constitucional debe garantizar pues constituye uno de los fines esenciales y propios del Estado que justifican su propia existencia ${ }^{18}$. De tal modo, si el servicio no funciona, o funciona tardiamente, queda atrapada la responsabilidad estatal por omision, lo cual cada vez mas se va a dar en el final de la decada por la destruccion de las herramientas con que el Estado presta sus funciones sociales indelegables (hospitales, instrumentales, etc.)

Lamentablemente la ficcion de la relacion de causalidad adecuada ${ }^{19}$ no nos

18. "Quien contrae contrae la obligacion de prestar un servicio - en el caso, de asistencia a la salud de la población-lo debe hacer en condiciones adecuadas para cumplir el fin en funcion del cual ha sido establecido y que es responsable de los perjuicios que causare su incumplimiento o su ejecucion irregular", Corte Suprema Justicia Diciembre 11-1994. Brescia Noem! L. \& Pcia. de Buenos Aires y otros s/ daños y perjuicios. Jurisprudencia Argentina 1997-II, sintesis.

19. Idem nota 15 , pag. 80 .

Revista da Faculdade de Direito da UFRGS, v. 13, 1997

permite avanzar en la verdadera causalidad condicionante ${ }^{20}$ que se eleva al umbral superior de los funcionarios que deben por la Constitucion Nacioal organizar adecuadamente el Servicio, vg. el Ministerio de Sa lud, y de esta forma el sistema logr" hacer eludir la verdadera responsabilidad.

Principios similares son aplicados en el ambito de los servicios privados. Resul ta interesante señalar que un sector importante de la doctrina extranjera ${ }^{21}$, siguiendo las corrientes mas modernas en materia de responsabilidad, considera al establecimiento asistencial privado como una empresa de servicios medicos, introduciendo e moderno concepto de empresa o ejercicio de una actividad de empresa, como un factor autónomo atributivo de responsabilidad.

El fundamento es que se trata de una actividad económica empresarial, de don de la responsabilidad del establecimiento asistencial resulta de la misma estructura económica y jurídica de su organización, lo que juridicamente se traduce en la imputación a su patrimonio de las actividades que realiza. El hecho de tratarse de una empresa que actúa con finalidad de lucro a traves de la prestacion medica que desarrolla, impone correlativamente la obligacion de cargar con los daños que con tal actividad produzca. 22

Esta tendencia objetivadora de la responsabilidad constituye un fenomeno mundial actual, mas acorde con los principios de equidad y proteccion a la victima, $y$ tanto la doctrina y jurisprudencia extranje$\mathrm{ra}^{23}$ han enfatizado sobre el derecho de los usuarios a un concreto funcionamiento del Hospital Publico en los terminos que acabamos de enunciar, y sobre el cual existe una copiosa jurisprudencia, especialmente en el derecho frances, denominada "faute de service".

20. Michel Foucoult, "Discurso, poder y subjetividad", pag. 46 y ss. Ed. El cielo por asalto, B.As. 1995.

21. Memeteau Gerard "La responsabilite civile medicale en droit compare francais et quebecois", Universite Mc. Gill, Montreal, 1990, pag. 120.

22. Ghersi Carlos A. La actividad económica como factor atributivo de responsabilidad, en obra "La responsabilidad". Homenaje al Dr. Isidoro Goldenberg, pag. 346, Ed. Abeledo Perrot, Bs. As. 1995.

23. idem nota 21 , pag. 119 